Proceedings of the Edinburgh Mathematical Society (2003) 46, 531-544 (C)

DOI:10.1017/S0013091502000974 Printed in the United Kingdom

\title{
COUNTABLE VERSUS UNCOUNTABLE RANKS IN INFINITE SEMIGROUPS OF TRANSFORMATIONS AND RELATIONS
}

\author{
P. M. HIGGINS ${ }^{1}$, J. M. HOWIE ${ }^{2}$, J. D. MITCHELL ${ }^{2}$ AND N. RUŠKUC ${ }^{2}$ \\ ${ }^{1}$ Department of Mathematics, University of Essex, \\ Colchester CO4 3SQ, UK (peteh@essex.ac.uk) \\ ${ }^{2}$ Mathematical Institute, University of St Andrews, \\ North Haugh, St Andrews, Fife KY16 9SS, UK \\ (jmh@st-and.ac.uk; mitchell@cii.fc.ul.pt; nik@mcs.st-and.ac.uk)
}

(Received 17 October 2002)

\begin{abstract}
The relative $\operatorname{rank} \operatorname{rank}(S: A)$ of a subset $A$ of a semigroup $S$ is the minimum cardinality of a set $B$ such that $\langle A \cup B\rangle=S$. It follows from a result of Sierpiński that, if $X$ is infinite, the relative rank of a subset of the full transformation semigroup $\mathcal{T}_{X}$ is either uncountable or at most 2 . A similar result holds for the semigroup $\mathcal{B}_{X}$ of binary relations on $X$.

A subset $S$ of $\mathcal{T}_{\mathbb{N}}$ is dominated (by $U$ ) if there exists a countable subset $U$ of $\mathcal{T}_{\mathbb{N}}$ with the property that for each $\sigma$ in $S$ there exists $\mu$ in $U$ such that $i \sigma \leqslant i \mu$ for all $i$ in $\mathbb{N}$. It is shown that every dominated subset of $\mathcal{T}_{\mathbb{N}}$ is of uncountable relative rank. As a consequence, the monoid of all contractions in $\mathcal{T}_{\mathbb{N}}$ (mappings $\alpha$ with the property that $|i \alpha-j \alpha| \leqslant|i-j|$ for all $i$ and $j$ ) is of uncountable relative rank.

It is shown (among other results) that $\operatorname{rank}\left(\mathcal{B}_{X}: \mathcal{T}_{X}\right)=1$ and that $\operatorname{rank}\left(\mathcal{B}_{X}: \mathcal{I}_{X}\right)=1$ (where $\mathcal{I}_{X}$ is the symmetric inverse semigroup on $X)$. By contrast, if $\mathcal{S}_{X}$ is the symmetric group, $\operatorname{rank}\left(\mathcal{B}_{X}: \mathcal{S}_{X}\right)=2$.
\end{abstract}

Keywords: transformation semigroups; rank; countable; binary relations

2000 Mathematics subject classification: Primary 20M20

\section{Introduction}

For a semigroup $S$ and a set $A \subseteq S$ the relative rank of $S$ modulo $A$ is the minimum cardinality of a set $B$ such that $A \cup B$ generates $S$; we denote this by $\operatorname{rank}(S: A)$. We also allow ourselves to call this the relative rank of $A$ in $S$. In [8] the authors showed that if we take $S=\mathcal{T}_{X}$, an infinite full transformation semigroup, then the relative rank of $S$ modulo $A$ is 2 in the cases where $A$ is either the full symmetric group or the set of all idempotents of $S$. On the other hand, if $A$ is itself a countable set and $A \cup B$ generates $\mathcal{T}_{X}$, then $B$ must be uncountable as $\mathcal{T}_{X}$ is itself uncountable. This prompts the question as to whether it is possible to find a subset $A$ of $\mathcal{T}_{X}$ which is of countable relative rank greater than 2. Surprisingly, the answer is 'no'. That conclusion can be deduced from Proposition 1.1, first proved by Sierpiński in 1935 [10]. A simpler proof was immediately furnished however by Banach [1]. Nonetheless, this result seems not to be widely known, 
as is evidenced by the fact that the often-quoted result of Evans [2], published in 1952, that any countable semigroup can be embedded in a two-generator semigroup, follows at once from Sierpiński's result. For that reason, and in order to make the paper more self-contained, we reproduce the short proof of Banach.

Throughout this paper, for a set $X, \mathcal{T}_{X}$ denotes the full transformation semigroup on $X, \mathcal{I}_{X}$ denotes the inverse semigroup of all partial bijections on $X, \mathcal{B}_{X}$ denotes the semigroup of all binary relations on $X$, and mappings and relations are written on the right. For standard definitions and more information see [7].

Proposition 1.1 (Sierpiński). Let $X$ be an infinite set. Then any countable subset $S$ of $\mathcal{T}_{X}$ is contained in a two-generated subsemigroup of $\mathcal{T}_{X}$.

Proof (Banach). Let the countably many members of $S$ be $\theta_{1}, \theta_{2}, \ldots$ Partition $X$ into a countable disjoint union of infinitely many sets $X_{0}, X_{1}, \ldots, X_{n}, \ldots$, all of the same cardinality as $X$, and similarly partition $X_{0}$ into $X_{0,1}, X_{0,2}, \ldots, X_{0, n}, \ldots$, again all of the same size as the parent set $X$.

Let $\beta \in \mathcal{T}_{X}$ be any mapping that maps $X_{n}$ bijectively onto $X_{n+1}$ for all $n \in \mathbb{N} \cup\{0\}$. Our second mapping $\gamma \in \mathcal{T}_{X}$ maps $X_{n}$ bijectively onto $X_{0, n}$ for all $n \geqslant 1$. Although we have yet to define $\gamma$ on $X_{0}$, we see that the mapping $\delta_{n}=\beta \gamma \beta^{n} \gamma$ is a well-defined bijection of $X$ onto $X_{0, n}$. We may therefore complete the definition of $\gamma$ by putting $x \delta_{n} \gamma=x \theta_{n},(x \in X)$. Since $\theta_{n}=\delta_{n} \gamma$ we obtain the factorization

$$
\theta_{n}=\beta \gamma \beta^{n} \gamma^{2} \quad(n \in \mathbb{N}),
$$

'et le théorème de M. Sierpiński est démontré'.

Corollary 1.2. The relative rank of a subset $S$ of $\mathcal{T}_{X}$, where $X$ is infinite, is either uncountable or at most 2 .

Also, since every semigroup $S$ embeds in $\mathcal{T}_{S^{1}}$, we immediately have the theorem of Evans [2].

Corollary 1.3. Every countable semigroup is embeddable in a two-generated semigroup.

Before continuing further we introduce the analogues of some standard properties of maps in terms of relations. We call a relation $\alpha \in \mathcal{B}_{X}$ injective if for every distinct pair $x, y \in \operatorname{dom}(\alpha)$ we have $x \alpha \cap y \alpha=\emptyset$. For $\beta \in \mathcal{B}_{X}$, if $\operatorname{im}(\beta)=X$ then we call the relation surjective. As in $[\mathbf{8}]$, where the infinite contraction index is used as a means of distinguishing permutations from maps which are not permutations, we require a property of relations that distinguish maps from relations which are not maps. To this end, for an arbitrary relation $\alpha \in \mathcal{B}_{X}$, we define the infinite expansion index to be the cardinality of the set

$$
P(\alpha)=\{x \in \operatorname{dom}(\alpha):|x \alpha|=|X|\},
$$

and we denote this cardinal by $p(\alpha)$. It is easy to see that $p(\alpha)=0$ for any $\alpha \in \mathcal{T}_{X}$. Further properties of relations are discussed in $\S 3$.

The analogue of Sierpiński's result for countable subsets of the semigroup of all binary relations can be proved using Banach's argument. 
Proposition 1.4. Any countable subset $S$ of the semigroup of all binary relations on $X$ is contained in a two-generated subsemigroup of $\mathcal{B}_{X}$.

Proof. The proof of this proposition is identical to the proof of Proposition 1.1. Note, however, that, since $\theta_{n} \in \mathcal{B}_{X}$, the definition

$$
x \delta_{n} \gamma=x \theta_{n} \quad(x \in X)
$$

makes $\gamma$, in general, not a function.

As in Corollary 1.2, we obtain the following.

Corollary 1.5. The relative rank of $\mathcal{B}_{X}$ modulo any subset $S$ is either uncountable or at most 2 .

It has also long been known that any countable group may be embedded in a twogenerated group [6]. In 1995 Fred Galvin explicitly proved the analogue of Sierpiński's result for groups when he showed in [3, Theorem 3.3] that every countable subset $A$ of the symmetric group $\mathcal{S}_{X}$ on an infinite base set $X$ is contained in a two-generated subgroup of $\mathcal{S}_{X}$. If the two group generators are $a$ and $b$, say, it follows immediately that $A$ is contained in the subsemigroup of $\mathcal{S}_{X}$ generated by the three elements $a, b$ and $a^{-1} b^{-1}$. Galvin goes on, however, to prove that the containing subsemigroup can also be taken to be two-generated as he shows how to ensure that the group generators $a$ and $b$ can have any prescribed orders $p$ and $2 q$, provided that $p$ is at least 3 and $q$ is at least 2 (see [3, Theorem 4.3]). The same paper contains the following result.

Proposition 1.6 (Theorem 5.8 in [3]). The relative rank of a subset $S$ of $\mathcal{S}_{X}$, where $X$ is infinite, is either uncountable or at most 1 .

In view of Corollaries 1.2 and 1.5 it is natural to ask for necessary and sufficient conditions for a subset $S$ to be of countable relative rank in $\mathcal{T}_{X}$ or $\mathcal{B}_{X}$. A sufficient condition is supplied by the following proposition.

Proposition 1.7. Suppose that $S$ is a subset of $\mathcal{T}_{X}$ (respectively, $\mathcal{B}_{X}$ ) and that there exist two disjoint subsets $Y, Z$ of $X$ each with the same cardinality as $X$ and such that

(Q) for every bijection $\theta$ from $Y$ to $Z$ there exists $\alpha \in S$ such that the restriction $\alpha \uparrow_{Y}=\theta$.

Then $\operatorname{rank}\left(\mathcal{T}_{X}: S\right) \leqslant 2$ (respectively, $\left.\operatorname{rank}\left(\mathcal{B}_{X}: S\right) \leqslant 2\right)$.

Proof. Let $S_{Y, Z}$ denote the set of all bijections from $Y$ to $Z$.

Let $S \subseteq \mathcal{T}_{X}$ be a set with the property (Q), and partition $Y$ into $Y_{1}, Y_{2}$ and $Z$ into $Z_{1}, Z_{2}$ with $\left|Y_{1}\right|=\left|Y_{2}\right|=\left|Z_{1}\right|=\left|Z_{2}\right|=|X|$. Let $\alpha$ be any bijection from $X$ to $Y_{1}$ and let $\beta \in \mathcal{T}_{X}$ be any mapping such that for all $x \in X$ the set $\left\{z \in Z_{1}: z \beta=x\right\}$ has cardinality $|X|$. Let $\gamma \in \mathcal{T}_{X}$ be arbitrary. Then there exists an injective mapping $\bar{\delta}$ from $Y_{1}$ to $Z_{1}$ such that $x \bar{\delta} \in x \alpha^{-1} \gamma \beta^{-1}$. We may extend $\bar{\delta}$ to an element $\delta$ of $S_{Y, Z}$. From the property (Q) we deduce that there exists $\epsilon \in S$ such that $\epsilon \uparrow_{Y}=\delta$. It follows that $\alpha \epsilon \beta=\alpha \delta \beta=\gamma$ and so $\mathcal{T}_{X}=\langle S, \alpha, \beta\rangle$. 
Let $S \subseteq \mathcal{B}_{X}$ have the property (Q). Let $\alpha \in \mathcal{B}_{X}$ be an injective relation with $P(\alpha)=X$ and $\operatorname{im}(\alpha)=Y_{1}$. Let $\beta$ be any partial mapping such that $\operatorname{dom}(\beta)=Z_{1}$ and for all $y \in X$ the set $y \beta^{-1}=\left\{z \in Z_{1}: z \beta=y\right\}$ has cardinality $|X|$. The sets $y \beta^{-1}$ are disjoint, since $\beta$ is a partial mapping, and each $y \beta^{-1}$ can be indexed by the set $X$ :

$$
y \beta^{-1}=\left\{z_{x, y}: x \in X\right\} .
$$

Thus $Z_{1}=\operatorname{dom}(\beta)$ can be indexed by $X \times X$ :

$$
Z_{1}=\left\{z_{x, y}: x, y \in X, z_{x, y} \beta=y\right\}
$$

and $z_{x, y}=z_{x^{\prime}, y^{\prime}}$ if and only if $x=x^{\prime}$ and $y=y^{\prime}$.

Consider an arbitrary $\gamma$ in $\mathcal{B}_{X}$. For each $x$ in $X$ denote $x \alpha$ by $D_{x}$, and let $E_{x}=$ $\left\{z_{x, y}: y \in x \gamma\right\}$. Since $\left|D_{x}\right|=|X|$ and $\left|E_{x}\right|=|x \gamma| \leqslant|X|$, there is a partial injection $\delta_{x}: D_{x} \rightarrow E_{x}$. (If $x \gamma=\emptyset$, that is, if $x \notin \operatorname{im}(\gamma)$, then $\delta_{x}$ is the empty mapping.) Since the sets $D_{x}(x \in X)$ and the sets $E_{x}(x \in X)$ are disjoint, the mapping $\delta^{\prime}=\bigcup_{x \in X} \delta_{x}$ is a partial injection from $Y_{1}$ into $Z_{1}$. Also, since $\left|Y \backslash \operatorname{dom}\left(\delta^{\prime}\right)\right|=Z \backslash \operatorname{im}\left(\delta^{\prime}\right)|=| X \mid$, we may extend $\delta^{\prime}$ to a bijection $\delta: Y \rightarrow Z$ in such a way that $\left(Y \backslash \operatorname{dom}\left(\delta^{\prime}\right)\right) \delta \subseteq Z_{2}$. Now, as before, let $\epsilon \in S$ be such that $\left.\epsilon\right|_{Y}=\delta$. Then, for each $x$ in $X$ we have that

$$
x \alpha \epsilon=x \alpha \delta=\left\{z_{x, y}: y \in x \gamma\right\} \cup F_{X},
$$

where $F_{X} \subseteq Z_{2}$. Hence $x \alpha \epsilon \beta=\left\{z_{x, y}: y \in x \gamma\right\} \beta=x \gamma$. Thus $\gamma=\alpha \epsilon \beta$, and so $\mathcal{B}_{X}=$ $\langle S, \alpha, \beta\rangle$ as required.

The next result shows that the property $(\mathrm{Q})$ is not a necessary condition for the countability of the relative rank.

Let $\mathbb{N}=\{1,2, \ldots\}$ taken in the natural order. A mapping $\alpha \in \mathcal{T}_{\mathbb{N}}$ is order preserving if it satisfies the condition that $i \leqslant j$ implies $i \alpha \leqslant j \alpha$ for all $i, j \in \mathbb{N}$. The set of all order-preserving mappings on $\mathbb{N}$ forms a subsemigroup $\mathcal{O}_{\mathbb{N}}$ of $\mathcal{T}_{\mathbb{N}}$.

Proposition 1.8. The semigroup $\mathcal{O}_{\mathbb{N}}$ is of relative rank 1 in $\mathcal{T}_{\mathbb{N}}$ and does not have the property $(Q)$ of Proposition 1.7.

Proof. Given any two equicardinal subsets $Y, Z$ of $\mathbb{N}$, there is a unique orderpreserving bijection from $Y$ onto $Z$, and so $\mathcal{O}_{\mathbb{N}}$ does not have the property $(\mathrm{Q})$.

Since $\mathcal{O}_{\mathbb{N}} \neq \mathcal{T}_{\mathbb{N}}$, the relative rank of $\mathcal{O}_{\mathbb{N}}$ is at least 1 . To show that it is exactly 1 , let $\gamma \in \mathcal{T}_{\mathbb{N}}$, and let $\delta$ be any mapping such that for each $n \in \mathbb{N}$ the set $\{i \in \mathbb{N}: i \delta=n\}$ is infinite. For each $k$ in $\mathbb{N}$, select an element $k \epsilon$ in $(k \gamma) \delta^{-1}$ in such a way that $k \epsilon<(k+1) \epsilon$ for all $k$. Then $\epsilon \in \mathcal{O}_{\mathbb{N}}$, and $\epsilon \delta=\gamma$. Thus $\left\langle\mathcal{O}_{\mathbb{N}}, \delta\right\rangle=\mathcal{T}_{\mathbb{N}}$.

For a semigroup $S$, two elements $x, y \in S$ are said to be $\mathcal{J}$-related if $S^{1} x S^{1}=\{u x v$ : $\left.u, v \in S^{1}\right\}=S^{1} y S^{1}$. It is easy to see that $\mathcal{J}$ is an equivalence relation. It is well known (see [7]) that two elements $\alpha$ and $\beta$ of $\mathcal{T}_{X}$ are $\mathcal{J}$-related if and only if $|\operatorname{im}(\alpha)|=|\operatorname{im}(\beta)|$. Let $J$ denote the set of all elements of $\mathcal{T}_{X}$ with image size equal to $|X|$. Since any factorization of a member $\alpha \in J$ involves only members of $J$ and since $J$ has the same cardinality as $\mathcal{T}_{X}$, it follows that the cardinality of the intersection with $J$ of any subsemigroup $S$ of 
$\mathcal{T}_{X}$ of countable relative rank must be equal to the cardinality of $\mathcal{T}_{X}$. In other words, $|S \cap J|=\left|\mathcal{T}_{X}\right|$. In fact the following stronger statement holds.

Proposition 1.9. If $S$ is a subset of $\mathcal{T}_{X}$ of countable relative rank, and if $U$ is any subset of $\mathcal{T}_{X}$ such that every injective restriction of an element of $S \cap J$ is also a restriction of an element of $U$, then $|U|=\left|\mathcal{T}_{X}\right|$.

Proof. Suppose that $A$ is a countable subset of $\mathcal{T}_{X}$ such that $\langle S \cup A\rangle=\mathcal{T}_{X}$. Take any $\pi \in \mathcal{S}_{X}$. Then $\pi=\theta_{1} \theta_{2} \cdots \theta_{n}$, where each $\theta_{i}$ lies in $S \cup A$. Since $\pi \in J$ it follows that $\theta_{i} \in J$ and $\theta_{i}$ is injective on the set $T=X \theta_{1} \ldots \theta_{i-1}$ (where the product is taken to be the identity mapping in the case $i=1$ ). By assumption, there exists $\xi_{i}$ in $U$ such that $\xi_{i} \uparrow_{T}=\theta_{i} \uparrow_{T}$, and so $\pi=\theta_{1} \cdots \theta_{i-1} \xi_{i} \theta_{i+1} \cdots \theta_{n}$. Replacing each $\theta_{i}$ in $S$ by an element $\xi_{i}$ of $U$, we express $\pi$ as a product of mappings from $U \cup A$. Thus $\mathcal{S}_{X} \subseteq\langle U \cup A\rangle$. Since $A$ is countable, and since $\left|\mathcal{S}_{X}\right|=\left|\mathcal{T}_{X}\right|>\aleph_{0}$, it follows that $|U|=\left|\mathcal{S}_{X}\right|=\left|\mathcal{T}_{X}\right|$.

Example 1.10. Let $X$ be the disjoint union of two infinite countable sets $Y$ and $Z$. Let $S$ be the subsemigroup

$$
S=\left\{\alpha \in \mathcal{T}_{X}:\left.\alpha\right|_{Z}=1_{Z}, Y \alpha \subseteq Y,|Y \alpha| \text { is finite }\right\}
$$

of $\mathcal{T}_{X}$, where $1_{Z}$ denotes the partial identity map with domain $Z$. Then $S \subseteq J$ and $S$ is uncountable. We shall show that $S$ is of uncountable relative rank in $\mathcal{T}_{X}$ by verifying that there exists a set $U$, as in Proposition 1.9, that is countable. Now the kernel transversals $T$ of members of $S$ each have the form $Z \cup Y^{\prime}$, where $Y^{\prime}$ is a finite subset of $Y$ and so the set of kernel transversals of members of $S$ is countable. Moreover, for each such kernel transversal $T$ there are only countably many mappings $\left.\alpha\right|_{T}(\alpha \in S)$. Hence there exists a countable subset $U$ of $\mathcal{T}_{X}$ such that every injective restriction of an element of $S$ is also a restriction of an element of $U$. Therefore, $S$ is of uncountable relative rank in $\mathcal{T}_{X}$ by Proposition 1.9.

The necessary condition on $S$ of Proposition 1.9 is not, however, sufficient for a subsemigroup $S$ of $\mathcal{T}_{X}$ to be of countable relative rank. This will follow from Proposition 2.4 of the next section.

\section{Dominated subsets}

We define a partial order ' $\leqslant$ ' on the elements of $\mathcal{T}_{\mathbb{N}}$ so that for any $\alpha, \beta \in \mathcal{T}_{\mathbb{N}}$ we write $\alpha \leqslant \beta$ if $i \alpha \leqslant i \beta$ for all $i \in \mathbb{N}$. For subsets $S, U \subseteq \mathcal{T}_{\mathbb{N}}$ we write $S \prec U$ if for each $\sigma \in S$ there exists $\mu \in U$ such that $\sigma \leqslant \mu$. The relation ' $\prec$ ' is not a partial order. It is a quasi-order, being reflexive and transitive, but, for example, if

$$
S=\left\{\alpha \in \mathcal{T}_{\mathbb{N}}: \operatorname{im}(\alpha)=2 \mathbb{N}\right\} \quad \text { and } \quad U=\left\{\alpha \in \mathcal{T}_{\mathbb{N}}: \operatorname{im}(\alpha)=2 \mathbb{N}+1\right\},
$$

then $S \prec U$ and $U \prec S$.

We say that a subset $S$ of $\mathcal{T}_{\mathbb{N}}$ is dominated (by $U$ ) if there exists a countable set $U \subseteq \mathcal{T}_{\mathbb{N}}$ such that $S \prec U$. Given $\sigma$ in $S$ and a function $\mu$ such that $\sigma \leqslant \mu$, we can always replace 
$\mu$ by $\mu^{\prime}$, defined inductively by

$$
1 \mu^{\prime}=1 \mu, \quad(n+1) \mu^{\prime}=\max \left\{n \mu^{\prime}+1,(n+1) \mu\right\} .
$$

Thus we may assume that every function in the dominating set $U$ is strictly monotonically increasing. We remark that $\mathcal{T}_{\mathbb{N}}$ itself is not dominated.

Theorem 2.1. Let $S_{1}, S_{2}, \ldots$ be a countable collection of subsets of $\mathcal{T}_{\mathbb{N}}$ dominated by the countable collection of sets $U_{1}, U_{2}, \ldots$, respectively. Then $\left\langle S_{1}, S_{2}, \ldots\right\rangle \prec\left\langle U_{1}, U_{2}, \ldots\right\rangle$ and $\left\langle S_{1}, S_{2}, \ldots\right\rangle$ is dominated.

Proof. It is clear that $S_{1} \cup S_{2} \cup \cdots=S \prec U=U_{1} \cup U_{2} \cup \cdots$. We show that $\langle S\rangle \prec\langle U\rangle$. Let $\alpha=\sigma_{1} \sigma_{2} \cdots \sigma_{n} \in\langle S\rangle$ and let $\kappa_{j} \in U$ be any function such that $\sigma_{j} \leqslant \kappa_{j}$ for each $j$. Then $\left(i \sigma_{1}\right) \sigma_{2} \leqslant\left(i \sigma_{1}\right) \kappa_{2} \leqslant\left(i \kappa_{1}\right) \kappa_{2}$ for all $i \in \mathbb{N}$. Hence by induction $i \alpha=i \sigma_{1} \sigma_{2} \cdots \sigma_{n} \leqslant$ $i \kappa_{1} \kappa_{2} \cdots \kappa_{n}$ for all $i \in \mathbb{N}$. It follows that $\langle S\rangle \prec\langle U\rangle$. Therefore, since $\langle U\rangle$ is countable, $\langle S\rangle$ is dominated.

Corollary 2.2. Any dominated subset $S$ of $\mathcal{T}_{\mathbb{N}}$ is of uncountable relative rank.

Proof. Let $U$ be any countable subset of $\mathcal{T}_{\mathbb{N}}$ such that $S \prec U$, and let $V$ denote an arbitrary countable subset of $\mathcal{T}_{\mathbb{N}}$. Then it is clear that $S \cup V \prec U \cup V$ and hence by Theorem 2.1 we have $\langle S \cup V\rangle \prec\langle U \cup V\rangle$, and $\langle S \cup V\rangle$ is dominated. Since $\mathcal{T}_{\mathbb{N}}$ is not dominated, it follows that $\langle S \cup V\rangle \neq \mathcal{T}_{\mathbb{N}}$ for every countable $V$.

Next, we introduce an equivalent characterization of dominated subsets.

Lemma 2.3. A subset $S \subseteq \mathcal{T}_{\mathbb{N}}$ is dominated if and only if there exists a function $\kappa \in \mathcal{T}_{\mathbb{N}}$ such that for all $\sigma \in S$ we have $i \sigma \leqslant i \kappa$ for all but finitely many $i \in \mathbb{N}$.

Proof. $(\Leftarrow)$ As observed above, we may assume that $\kappa$ is strictly increasing. We begin by observing that for each $\alpha \in S$ there exists $M_{\alpha} \in \mathbb{N}$ such that for any $j \leqslant M_{\alpha}$ we have $j \alpha \leqslant M_{\alpha} \kappa$. Indeed, there are only finitely many $i \in \mathbb{N}$ such that $i \alpha>i \kappa$, and so we may find the maximum such $i \alpha$, which we denote by $N$. Since $\kappa$ is monotonic increasing there exists $M_{\alpha}$ such that $M_{\alpha} \kappa \geqslant N$, and it is easy to see that this $M_{\alpha}$ has the desired properties. We define a countable set of functions $K=\left\{\kappa_{i}: i \in \mathbb{N}\right\}$ as follows:

$$
n \kappa_{i}= \begin{cases}n \kappa & n \geqslant i, \\ i \kappa & n<i .\end{cases}
$$

Then for arbitrary $\alpha \in S$ we have $\alpha \leqslant \kappa_{M_{\alpha}}$, and hence $S \prec K$.

$(\Rightarrow)$ Let $\left\{\kappa_{i}: i \in \mathbb{N}\right\}$ denote any set which dominates $S$. Define

$$
n \kappa=\sum_{i=1}^{n} n \kappa_{i} .
$$

Then for any $\sigma \in S$ there exists $\kappa_{r}$ such that $n \sigma \leqslant n \kappa_{r}$ for all $n \in \mathbb{N}$. Hence for $n \geqslant r$ we have

$$
n \kappa=\sum_{i=1}^{n} n \kappa_{i} \geqslant n \kappa_{r} \geqslant n \sigma
$$

and hence $\kappa$ has the required property. 
We shall refer to a function with the properties of $\kappa$ in the above proof as a dominating function.

A mapping $\alpha \in \mathcal{T}_{\mathbb{N}}$ is called a contraction if it satisfies the condition that $|i \alpha-j \alpha| \leqslant$ $|i-j|$ for all $i, j \in \mathbb{N}$. Equivalently, $\alpha$ is a contraction if it maps intervals to intervals. Let $\mathcal{C}$ denote the semigroup of all contractions in $\mathcal{T}_{\mathbb{N}}$. We now show that $\mathcal{C}$ satisfies the condition of Proposition 1.9. To see this, let $M=\{1,3,5,7, \ldots\}$. We show that there are $\left|\mathcal{T}_{\mathbb{N}}\right|$ injections from $M$ into $\mathbb{N}$ which are restrictions of mappings in $\mathcal{C} \cap J$. Let $\sigma=\left(\epsilon_{1}, \epsilon_{2}, \ldots\right)$ be a sequence of 0 s and 1 s. Define $\alpha_{\sigma}: M \rightarrow \mathbb{N}$ by

$$
1 \alpha_{\sigma}=1, \quad(2 i+1) \alpha_{\sigma}= \begin{cases}(2 i-1) \alpha_{\sigma}+1 & \text { if } \epsilon_{i}=0 \\ (2 i-1) \alpha_{\sigma}+2 & \text { if } \epsilon_{i}=1\end{cases}
$$

Clearly, there are $2^{|\mathbb{N}|}=\left|\mathcal{T}_{\mathbb{N}}\right|$ mappings $\alpha_{\sigma}$. On the other hand, $\alpha_{\sigma}=\beta_{\sigma} \uparrow_{M}$, where $\beta_{\sigma} \in \mathcal{C} \cap J$ is defined by

$$
(2 i-1) \beta_{\sigma}=(2 i-1) \alpha_{\sigma}, \quad(2 i) \beta_{\sigma}=(2 i-1) \alpha_{\sigma}+1 .
$$

Despite this we have the following proposition.

Proposition 2.4. The monoid $\mathcal{C}$ of all contractions in $\mathcal{T}_{\mathbb{N}}$ is of uncountable relative rank.

Proof. By Corollary 2.2 and Lemma 2.3 it remains only to observe that $\mathcal{C}$ has a dominating function $\kappa$ where $n \kappa=n^{2}$. This follows from the definition of a contraction, since for any $\alpha \in \mathcal{C}$ we have $n \alpha \leqslant 1 \alpha+(n-1) \leqslant n^{2}$ for all sufficiently large $n$.

As introduced above, the notion of a dominated subset $S$ of $\mathcal{T}_{\mathbb{N}}$ is only with respect to the natural well-ordering of $\mathbb{N}$. However, we note that it is possible to show that the property of being a dominated subset is maintained if we take another well-ordering of $\mathbb{N}$ which is isomorphic to the natural ordering.

Theorem 2.5. If a subset $S$ of $\mathcal{T}_{\mathbb{N}}$ is dominated with respect to the natural wellordering of $\mathbb{N}$, then $S$ is dominated with respect to every isomorphic well-ordering of $\mathbb{N}$.

Proof. We assume that $S$ is dominated by the set $U=\left\{\kappa_{i}: i \in \mathbb{N}\right\}$ with respect to the standard ordering ' $\leqslant$ ' on the natural numbers. Let us take another well-ordering ' $\leqslant \pi$ ' determined by some permutation $\pi$ of $\mathbb{N}$ in that the ordering ' $\leqslant \pi$ ' is given by

$$
1 \pi \leqslant_{\pi} 2 \pi \leqslant_{\pi} \cdots \leqslant_{\pi} n \pi \leqslant_{\pi} \cdots .
$$

Define a function $\epsilon$ such that

$$
n \epsilon=\sup _{\leqslant \pi}\left\{m: m \leqslant \max _{\leqslant}\left\{n \kappa_{i}: i \leqslant n\right\}\right\} .
$$

Then for any $\alpha \in S$ we have $i \alpha \leqslant i \kappa_{r}$ for all $i \in \mathbb{N}$ and for some $r \in \mathbb{N}$. In particular, $r \alpha \leqslant r \kappa_{r}$ and so for $n \geqslant r$ we have

$$
n \alpha \leqslant n \kappa_{r} \leqslant \max _{\leqslant}\left\{n \kappa_{i}: i \leqslant n\right\} .
$$

Hence $n \alpha \leqslant \pi n \epsilon$. Thus $\epsilon$ is a dominating function for $S$. 
Example 2.6. Let $E \subseteq \mathcal{T}_{\mathbb{N}}$ denote the set of all functions $\alpha$ in $\mathcal{T}_{\mathbb{N}}$ such that $i \alpha$ is an even number, for every $i \in \mathbb{N}$. This set is clearly not dominated in $\mathcal{T}_{\mathbb{N}}$, since any candidate $\kappa$ for a dominating function fails to dominate $2 \kappa \in E$. We see, however, that $E$ is dominated with respect to a different (and non-isomorphic) well-ordering of $\mathbb{N}$. Define an order so that every even number lies below every odd number, and the natural order is maintained within the set of all even and the set of all odd numbers. It is clear that the function $\alpha \in \mathcal{T}_{\mathbb{N}}$, defined by $n \alpha=1$, dominates $E$ with this well-ordering.

Remark 2.7. We note that, if the original ordering of $\mathbb{N}$ in Theorem 2.5 is not taken to be the natural well-ordering (or a well-ordering isomorphic to the natural well-ordering), then the result does not necessarily hold. To see this, we define a well-ordering on $\mathbb{N}$ such that every even number lies above every odd number. This well-ordering is isomorphic to the order in the previous example, but the set $E$ is not dominated with respect to this ordering.

\section{Binary relations}

We now consider some of the problems from above and from $[\mathbf{8}]$ in a more general setting. Throughout the following, let $X$ be an infinite set and let $\mathcal{B}_{X}$ denote the semigroup of all binary relations on $X$.

We note that the argument used to prove both Sierpiński's result and its generalization to the semigroup of all binary relations can equally well be used to prove the analogous result for partial maps. For completeness, we state this result.

Proposition 3.1. Let $Y$ be any infinite subset of $X$ and let $S$ denote the subsemigroup consisting of all elements in $\mathcal{T}_{X}$ which fix $X \backslash Y$ pointwise. Then any countable subset of $S$ is contained in a two-generated subsemigroup of $S$. In particular, the relative rank of $S$ modulo any subset is either uncountable or at most 2 .

In the case that $Y=\emptyset$ we obtain Proposition 1.1, and in the case that $|X \backslash Y|=1$ we have the following corollary.

Corollary 3.2. Any countable subset of the partial transformation semigroup $\mathcal{P}_{X}$ is contained in a two-generated subsemigroup of $\mathcal{P}_{X}$. In particular, the relative rank of $\mathcal{P}_{X}$ modulo any subset is either uncountable or at most 2.

We now consider the relative rank of $\mathcal{B}_{X}$ modulo some standard subsemigroups, simulating the results in $[\mathbf{8}]$. We begin by finding the relative rank of $\mathcal{B}_{X}$ modulo the set of all injective mappings in $\mathcal{T}_{X}$.

Theorem 3.3. The relative rank of $\mathcal{B}_{X}$ modulo $\mathcal{T}_{X} \cap \mathcal{I}_{X}$ is 1 .

Proof. Partition $X$ into three disjoint subsets $Y, Z$ and $W$, with $|Y|=|Z|=|W|=$ $|X|$. Similarly, partition $Y$ and $Z$ into disjoint subsets $Y_{x}$ and $Z_{x}(x \in X)$, again with $\left|Y_{x}\right|=\left|Z_{x}\right|=|X|$. Let $\rho \in \mathcal{T}_{X} \cap \mathcal{I}_{X}$ be any bijection from $X$ to $Y$, and for all $x \in X$ let $\delta_{x}$ be any bijection from $Z_{x}$ to $X$. Define $\Delta \in \mathcal{B}_{X}$ by

$$
x \Delta= \begin{cases}Y_{x \rho^{-1}} & x \in Y, \\ x \delta_{y} & x \in Z_{y}(y \in X) .\end{cases}
$$


Let $\alpha \in \mathcal{B}_{X}$ be arbitrary. Let $\sigma \in \mathcal{T}_{X} \cap \mathcal{I}_{X}$ be any mapping which takes $|x \alpha|$ elements of $Y_{x}$ injectively to $x \alpha \delta_{x}^{-1}\left(\subseteq Z_{x}\right)$ for all $x \in \operatorname{dom}(\alpha)$ and which takes the remaining elements of $X$ to $W$ injectively. For $x \in \operatorname{dom}(\alpha)$,

$$
x \rho \Delta \sigma \Delta=Y_{x} \sigma \Delta=\left(x \alpha \delta_{x}^{-1}\right) \Delta=x \alpha \delta_{x}^{-1} \delta_{x}=x \alpha,
$$

and for $x \notin \operatorname{dom}(\alpha)$ we have $x \rho \Delta \sigma \subseteq W$ and so $x \rho \Delta \sigma \Delta$ is undefined. We have shown that $\alpha \in\left\langle\mathcal{T}_{X} \cap \mathcal{I}_{X}, \Delta\right\rangle$ and so $\mathcal{B}_{X}=\left\langle\mathcal{T}_{X} \cap \mathcal{I}_{X}, \Delta\right\rangle$.

Corollary 3.4. The relative rank of $\mathcal{B}_{X}$ modulo $\mathcal{T}_{X}$ or $\mathcal{I}_{X}$ is 1 .

Recall that a cardinal $\kappa$ is singular if there exist sets $Y$ and $Z_{y}$ for $y \in Y$ such that $|Y|<\kappa$ and $\left|Z_{y}\right|<\kappa$ but

$$
\left|\bigcup_{y \in Y} Z_{y}\right|=\kappa .
$$

A cardinal is called regular if it is not singular. For more details about singular and regular cardinals see $[\mathbf{5}]$ or $[\mathbf{9}]$.

Next we prove the main result in this section, which is a characterization of all subsets $U$ of $\mathcal{B}_{X}$ such that $\left\langle\mathcal{T}_{X} \cup U\right\rangle=\mathcal{B}_{X}$, in the case where $|X|$ is regular.

Theorem 3.5. Let $|X|$ be a regular cardinal and let $U \subseteq \mathcal{B}_{X}$ be arbitrary. Then $\mathcal{T}_{X} \cup U$ generates $\mathcal{B}_{X}$ if and only if $U$ contains relations $\alpha$ and $\beta$ satisfying

(i) there exists $Y \subseteq P(\alpha)$ such that $|Y|=|X|$ and

$$
\left|y_{0} \alpha \backslash \bigcup_{\substack{y \in Y \\ y \neq y_{0}}} y \alpha\right|=|X|,
$$

for every $y_{0} \in Y$;

(ii) $\operatorname{dom}(\beta) \subsetneq X$;

(iii) there exists $Z \subseteq \operatorname{dom}(\beta)$ such that $|Z|=|X|$ and the sets $z \beta(z \in Z)$ are pairwise disjoint.

Proof. $(\Leftarrow)$ We prove the converse implication by showing that every element of $\mathcal{B}_{X}$ can be written as a product of elements of $\mathcal{T}_{X}, \alpha$ and $\beta$. In fact, we prove that every relation $\gamma \in \mathcal{B}_{X}$ has a factorization $\gamma=\rho \alpha \sigma \beta \pi$, where $\rho, \sigma, \pi \in \mathcal{T}_{X}$.

To see this, let $\rho$ be any bijection from $X$ to $Y$. Partition $Z$ into disjoint sets $Z_{x}$ $(x \in X)$ each of cardinality $|X|$. Let $\sigma$ be any mapping which takes

$$
y_{0} \alpha \backslash\left(\bigcup_{y \in Y \backslash\left\{y_{0}\right\}} y \alpha\right)
$$


to $Z_{y_{0} \rho^{-1}}$ injectively for each $y_{0} \in Y$ such that $y_{0} \rho^{-1} \in \operatorname{dom}(\gamma)$, and which takes everything else to a fixed element $x_{0} \in X \backslash \operatorname{dom}(\beta)$. Finally, let $\pi \in \mathcal{T}_{X}$ be any mapping such that $\left(Z_{x} \beta\right) \pi=x \gamma$ for every $x \in \operatorname{dom}(\gamma)$. Then for $x \in \operatorname{dom}(\gamma)$ we have

$$
x \rho \alpha \sigma \beta \pi=\left(x \rho \alpha \backslash \bigcup_{\substack{y \in Y \\ y \neq x \rho}} y \alpha\right) \sigma \beta \pi=Z_{x} \beta \pi=x \gamma .
$$

It is clear that $x \rho \alpha \sigma \beta \pi$ is not defined for $x \notin \operatorname{dom}(\gamma)$.

$\Rightarrow$ We prove the direct implication using the following two lemmas. Let $S_{1}$ denote the set of all relations that fail to satisfy at least one of (ii) and (iii), and let $S_{2}$ denote the set of all relations not satisfying (i).

Lemma 3.6. The set $S_{1}$ is a proper subsemigroup of $\mathcal{B}_{X}$ containing $\mathcal{T}_{X}$ but not $\mathcal{P}_{X}$.

Proof. We see that $\mathcal{T}_{X} \subseteq S_{1}$ since no element of $\mathcal{T}_{X}$ satisfies (ii). Any proper partial identity map satisfies both (ii) and (iii) and so $\mathcal{P}_{X}$ is not contained in $S_{1}$.

We show that $S_{1}$ is a semigroup by demonstrating that

$$
\alpha \beta \notin S_{1} \quad \text { and } \beta \in S_{1} \quad \Rightarrow \quad \alpha \notin S_{1} .
$$

So suppose that $\alpha \beta$ satisfies (ii) and (iii), and that $\beta$ fails to satisfy at least one of (ii) and (iii). Let $Z$ be such that $Z \subseteq \operatorname{dom}(\alpha \beta),|Z|=|X|$ and the sets $z \alpha \beta(z \in Z)$ are non-empty and disjoint. For each $z$ in $Z$ we may choose $t_{z}$ in $z \alpha$ such that $t_{z} \in \operatorname{dom}(\beta)$. The elements $t_{z}(z \in Z)$ are all distinct: if $t_{z}=t_{w}(w, z \in Z, w \neq z)$, then $t_{z} \beta \subseteq z \alpha \beta$, $t_{w} \beta \subseteq w \alpha \beta$ and so $z \alpha \beta \cap w \alpha \beta \neq \emptyset$, a contradiction. Thus $T=\left\{t_{z}: z \in Z\right\} \subseteq \operatorname{dom}(\beta)$, and $|T|=|Z|=|X|$. Also, since $t_{z} \beta \subseteq z \alpha \beta$ for all $z$, the sets $t_{z} \beta$ are disjoint.

Thus $\beta$ satisfies (iii), and so, by our assumptions, does not satisfy (ii). That is, $\operatorname{dom}(\beta)=X$. It now follows that $\alpha$ satisfies (iii), since there exists $Z \subseteq \operatorname{dom}(\alpha \beta) \subseteq$ $\operatorname{dom}(\alpha)$ such that $|Z|=|X|$, and certainly the sets $z \alpha(z \in Z)$ are disjoint. Since $\operatorname{dom}(\alpha \beta) \subsetneq X$ and $\operatorname{dom}(\beta)=X$, it follows that $\operatorname{dom}(\alpha) \subsetneq X$, and so $\alpha \notin S_{1}$.

Lemma 3.7. If $|X|$ is a regular cardinal, then $S_{2}$ is a proper subsemigroup of $\mathcal{B}_{X}$ containing $\mathcal{P}_{X}$.

Proof. That $\mathcal{P}_{X} \subseteq S_{2}$ is obvious since no mapping satisfies (i).

Suppose now that $\alpha \beta$ satisfies (i). We show that either $\alpha$ or $\beta$ satisfies (i). There exist disjoint sets $Z_{x} \subseteq x \alpha \beta(x \in Y)$ such that $\left|Z_{x}\right|=|X|$. Let $Y_{x}=x \alpha \cap Z_{x} \beta^{-1}$, it is easy to see that these sets are disjoint. If $|X|$ of the sets $Y_{x}$ have cardinality $|X|$ then $\alpha$ satisfies (i). Otherwise, $\beta$ satisfies (i).

It follows from these two results that if $U$ contains no element satisfying (i), then $\left\langle\mathcal{T}_{X} \cup U\right\rangle \subseteq S_{2} \subsetneq \mathcal{B}_{X}$, or if $U$ contains no element satisfying (ii) and (iii), then $\left\langle\mathcal{T}_{X} \cup U\right\rangle \subseteq$ $S_{1} \subsetneq \mathcal{B}_{X}$, and the result follows. 
This proof does not explicitly rely on the fact that the maps we are considering are full maps. We may easily modify this result so that it holds for partial maps as well. Here we do not need a mapping $\beta$ satisfying (ii) and (iii). Instead, in the $(\Leftarrow)$ part of the proof we can use a partial map $\xi$ which maps

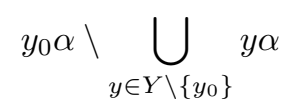

onto $y_{0} \rho^{-1} \gamma$ and is otherwise undefined, to express an arbitrary $\gamma$ in $\mathcal{B}_{X}$ as $\rho \alpha \xi$. Thus we have the following corollary.

Corollary 3.8. The relative rank of $\mathcal{B}_{X}$ modulo $\mathcal{P}_{X}$ is 1 . Furthermore, if $|X|$ is a regular cardinal and $U \subseteq \mathcal{B}_{X}$ is arbitrary, then $\mathcal{P}_{X} \cup U$ generates $\mathcal{B}_{X}$ if and only if $U$ contains a relation which satisfies (i) in the previous theorem.

It follows from Proposition 1.7 that the relative rank of $\mathcal{B}_{X}$ modulo both the symmetric group $\mathcal{S}_{X}$ or the set of idempotent relations $\mathcal{B E}_{X}$ is less than or equal to 2 . Indeed, if we partition $X$ into disjoint sets $Y$ and $Z$, with $|Y|=|Z|$, then for any bijection $\beta$ from $Y$ to $Z$ the mapping $\alpha \in \mathcal{T}_{X}$ defined by

$$
x \alpha= \begin{cases}x \beta & x \in Y, \\ x & x \in Z,\end{cases}
$$

is an idempotent with $\alpha \uparrow_{Y}=\beta$. That $\operatorname{rank}\left(\mathcal{B}_{X}: \mathcal{S}_{X}\right) \leqslant 2$ and $\operatorname{rank}\left(\mathcal{B}_{X}: \mathcal{B} \mathcal{E}_{X} \cap \mathcal{T}_{X}\right) \leqslant 2$ also follows from Corollary 3.4 and [8, Theorem 3.3]. We show, in both cases, that these bounds are sharp.

Theorem 3.9. The relative rank of $\mathcal{B}_{X}$ modulo the symmetric group $\mathcal{S}_{X}$ is 2 .

Proof. Observe that $\mathcal{B}_{X}$ is the union of the semigroups $T_{1}=\left\{\alpha \in \mathcal{B}_{X}: \operatorname{dom}(\alpha)=X\right\}$ and $T_{2}=\left\{\alpha \in \mathcal{B}_{X}: \operatorname{dom}(\alpha) \subsetneq X\right\} \cup \mathcal{S}_{X}$. Now, if $\left\langle\mathcal{S}_{X}, \mu\right\rangle=U$, then $\mu \in T_{1}$ or $\mu \in T_{2}$, and so either $U \subseteq T_{1} \subsetneq \mathcal{B}_{X}$ or $U \subseteq T_{2} \subsetneq \mathcal{B}_{X}$. Hence $\operatorname{rank}\left(\mathcal{B}_{X}: \mathcal{S}_{X}\right)>1$.

A similar argument can be applied to the set of all full surjective mappings to give the following corollary.

Corollary 3.10. The relative rank of $\mathcal{B}_{X}$ modulo the set of all surjective mappings in $\mathcal{T}_{X}$ is 2 .

We now consider the set $\mathcal{B E}_{X}$ of all idempotent relations in $\mathcal{B}_{X}$. In order to prove that the bound given above is sharp we require the following lemmas.

Lemma 3.11. If $\alpha \beta \in \mathcal{S}_{X}$ and $\alpha^{2}=\alpha$, then $\alpha=1_{X}$. Likewise, if $\beta^{2}=\beta$, then $\beta=1_{X}$.

Proof. Suppose that there exist $x, y$ in $X$ such that $x \neq y$ and $(x, y) \in \alpha$. Since $\alpha \beta \in \mathcal{S}_{X}$, there exist $z, t \in X$ such that $(y, z) \in \alpha,(z, t) \in \beta$. From $(x, y) \in \alpha$ and $(y, z) \in \alpha$ we deduce that $(x, z) \in \alpha^{2}=\alpha$. But then $t \in x \alpha \beta \cap y \alpha \beta$, a contradiction. To prove the second half, observe that $\left(\beta^{-1}\right)^{2}=\beta^{-1}$ and $\beta^{-1} \alpha^{-1} \in \mathcal{S}_{X}$. 
Lemma 3.12. For $\alpha, \beta \in \mathcal{B}_{X}$, if $\alpha \beta \alpha \in \mathcal{S}_{X}$, then $\beta \in \mathcal{S}_{X}$.

Proof. Clearly, $\alpha$ is surjective, $\operatorname{dom}(\alpha)=X$ and $\alpha \Upsilon_{\mathrm{im}(\alpha \beta)}$ is a surjective map. For each $x \in \operatorname{im}(\alpha \beta)$ there is exactly one element in $x \alpha$, since $\alpha \beta \alpha$ is a map. Now, $x \in \operatorname{dom}(\alpha \beta \alpha)(=X)$ and so $x \alpha \in \operatorname{dom}(\beta \alpha)$ for all $x \in \operatorname{im}(\alpha \beta)$. Therefore, $X=\operatorname{im}\left(\alpha \uparrow_{\operatorname{im}(\alpha \beta)}\right) \subseteq \operatorname{dom}(\beta \alpha)$, which implies that $X \subseteq \operatorname{dom}(\beta)$. It follows that $\alpha$ is injective, for if $x \neq y$, then $t \in x \alpha \cap y \alpha$ implies that $t \beta \alpha \subseteq x \alpha \beta \alpha \cap y \alpha \beta \alpha$, a contradiction.

The same argument applied to $\alpha^{-1} \beta^{-1} \alpha^{-1}$ yields that $\alpha^{-1}$ is injective, which is equivalent to $\alpha$ being a partial mapping. Hence $\alpha \in \mathcal{S}_{X}$, and from this it easily follows that $\beta \in \mathcal{S}_{X}$, as required.

We use these two lemmas to prove the following theorem.

Theorem 3.13. The relative rank of $\mathcal{B}_{X}$ modulo the set of idempotent relations $\mathcal{B E}_{X}$ or modulo $\mathcal{T E}_{X}\left(=\mathcal{B E}_{X} \cap \mathcal{T}_{X}\right)$ is 2 .

Proof. Suppose that $\left\langle\mathcal{B E}_{X}, \mu\right\rangle=\mathcal{B}_{X}$ for some $\mu \in \mathcal{B}_{X}$ and let $\pi \in \mathcal{S}_{X}$. We may write $\pi=\gamma_{1} \gamma_{2} \cdots \gamma_{n}$, where $\gamma_{i} \in \mathcal{B E}_{X} \cup\{\mu\}$. We proceed to show, by induction on $n$, that $\gamma_{i} \in\left\{1_{X}, \mu\right\}$ for each $i$. By Lemma 3.11 we may assume that $\gamma_{1}=\gamma_{n}=\mu$. Hence in the case that $n=1$, or 2 , our hypothesis is verified. For $n>2$ we have $\gamma_{2} \cdots \gamma_{n-1} \in \mathcal{S}_{X}$ by Lemma 3.12, and by induction $\gamma_{2}, \ldots, \gamma_{n-1} \in\left\{1_{X}, \mu\right\}$, as required.

\section{Inverse semigroups}

We now consider many of the same questions for the symmetric inverse semigroup $\mathcal{I}_{X}$ as we have so far considered for the semigroup of binary relations and the full transformation semigroup.

Remark 4.1. In this section, we shall consider a different definition of relative rank. For a subset $A$ of an inverse semigroup $S$ we define the relative rank of $S$ modulo $A$ to be the minimum cardinality of any set $B \subseteq S$ such that the inverse subsemigroup generated by $A \cup B$ equals $S$. The important point to note is that we may use the inverses of elements of $A \cup B$.

The first important question to answer is whether every countable inverse subsemigroup is contained in a two-generated inverse subsemigroup of $\mathcal{I}_{X}$. We shall show that this is, in fact, the case. Following from this, and more importantly for our purposes here, we show that the relative rank of every inverse subsemigroup of $\mathcal{I}_{X}$ is uncountable or at most 2. A more interesting question is whether, or not, the relative rank of every inverse subsemigroup of $\mathcal{I}_{X}$ is uncountable or at most 1 (as is the case with groups).

Proposition 4.2. Any countable subset $S$ of the symmetric inverse semigroup on $X$ is contained in a two-generated inverse subsemigroup of $\mathcal{I}_{X}$.

Proof. The proof is similar to the proof of Sierpiński's result, the essential difference being that here we are dealing with inverse subsemigroups and so allow ourselves to use inverses of generators. 
Let the countably many members of $S$ be $\alpha_{1}, \alpha_{2}, \ldots$ Partition $X$ into a countable disjoint union of infinitely many sets $X_{0}, X_{1}, \ldots, X_{n}, \ldots$, all of the same cardinality as $X$, and similarly partition $X_{0}$ into $X_{0,1}, X_{0,2}, \ldots, X_{0, n}, \ldots$, again all of the same size as the set $X$.

Let $\beta \in \mathcal{I}_{X}$ be any partial bijection that maps $X_{n}$ bijectively onto $X_{n+1}$ for all $n=$ $0,1,2, \ldots$ We have $\operatorname{dom}(\beta)=X$ and $\operatorname{im}(\beta)=X \backslash X_{0}$.

Our second partial bijection $\gamma \in \mathcal{I}_{X}$ maps $X_{n}$ bijectively onto $X_{0, n}$ for all $n \geqslant 1$. We see that $\beta \gamma \beta^{n}$ is a well-defined bijection from $X$ onto $X_{n}$, and so $\delta_{n}=\beta \gamma \beta^{n} \gamma$ is a well-defined bijection from $X$ to $X_{0, n}$.

So far, $\gamma$ is defined on $X \backslash X_{0}$ with its image lying in $X_{0}$. We may therefore complete the definition of $\gamma$ by defining $x \delta_{n} \gamma=x \alpha_{n} \beta \gamma \beta^{n} \in X_{n}(n \geqslant 1)$ for all $x \in X$.

Since $\alpha_{n}=\delta_{n} \gamma \beta^{-n} \gamma^{-1} \beta^{-1}$, we obtain the factorization

$$
\alpha_{n}=\beta \gamma \beta^{n} \gamma^{2} \beta^{-n} \gamma^{-1} \beta^{-1} \quad(n=1,2, \ldots),
$$

and so $S$ is contained in the inverse subsemigroup generated by $\beta$ and $\gamma$.

Again, using the same argument as Corollary 1.2, we obtain the following corollary.

Corollary 4.3. The relative rank of a subset $S$ of $\mathcal{I}_{X}$, where $X$ is infinite, is either uncountable or at most 2.

A consequence of Proposition 4.2 is the well-known result that any countable inverse semigroup can be embedded in a two-generated inverse semigroup, attributed in [4] to C. J. Ash.

We now consider the relative rank of the symmetric inverse semigroup modulo some of the standard inverse subsemigroups. We require the notion of the defect of a map $\alpha$, which is simply the cardinality of the complement of the image of the map: $d(\alpha)=|X \backslash \operatorname{im}(\alpha)|$.

Lemma 4.4. If $\alpha, \beta \in \mathcal{I}_{X}$, then $d(\alpha \beta)=d(\alpha)+d(\beta)$.

For details see [8, Lemma 2.1(i)].

Theorem 4.5. The relative rank of $\mathcal{I}_{X}$ modulo $\mathcal{S}_{X}$ is 1 . Furthermore, for $\mu \in \mathcal{I}_{X}$ the set $\mathcal{S}_{X} \cup\{\mu\}$ generates $\mathcal{I}_{X}$ (as an inverse semigroup) if and only if $\left\{d(\mu), d\left(\mu^{-1}\right)\right\}=$ $\{0,|X|\}$.

Proof. $(\Rightarrow)$ Let $S_{1}=\left\{\alpha \in \mathcal{I}_{X}: d(\alpha)<|X|\right.$ and $\left.d\left(\alpha^{-1}\right)<|X|\right\}$ and let $S_{2}=I \cup \mathcal{S}_{X}$, where $I=\left\{\alpha \in \mathcal{I}_{X}: d(\alpha)>0\right.$ and $\left.d\left(\alpha^{-1}\right)>0\right\}$. It follows from Lemma 4.4 that both $S_{1}$ and $S_{2}$ are proper subsemigroups of $\mathcal{I}_{X}$. Now, if $\mathcal{I}_{X}=\left\langle\mathcal{S}_{X}, \mu\right\rangle$, for some $\mu \in \mathcal{I}_{X}$, then $\mu \notin S_{1}$ and $\mu \notin S_{2}$. Since $\mu \notin S_{1}$, either $d(\mu)=|X|$ or $d\left(\mu^{-1}\right)=|X|$. Since $\mu \notin S_{2}$, in the former case $d\left(\mu^{-1}\right)=0$ and in the latter case $d(\mu)=0$, as required.

$(\Leftarrow)$ Assume, without loss of generality, that $d(\mu)=|X|$ and $\operatorname{dom}(\mu)=X$. Let $\alpha \in \mathcal{I}_{X}$ be arbitrary. Let $\bar{\pi}=\mu^{-1} \alpha \mu$. Then $\operatorname{dom}(\bar{\pi}) \subseteq \operatorname{dom}\left(\mu^{-1}\right)=\operatorname{im}(\mu)$, and so $|X \backslash \operatorname{dom}(\bar{\pi})| \geqslant$ $|X \backslash \operatorname{im}(\mu)|=|X|$. Also $\operatorname{im}(\bar{\pi}) \subseteq \operatorname{im}(\mu)$, which implies $|X \backslash \operatorname{im}(\bar{\pi})| \geqslant|X \backslash \operatorname{im}(\mu)|=|X|$. We extend $\bar{\pi}$ to $\pi \in \mathcal{S}_{X}$ so that

$$
(\operatorname{im}(\mu) \backslash \operatorname{dom}(\alpha) \mu) \pi \subseteq X \backslash \operatorname{im}(\mu)
$$


we can do this because $|X \backslash \operatorname{im}(\mu)|=d(\mu)=|X|$. For $x \in \operatorname{dom}(\alpha)$ we have

$$
x \mu \pi \mu^{-1}=x \mu \bar{\pi} \mu^{-1}=x \mu \mu^{-1} \alpha \mu \mu^{-1}=x \alpha .
$$

On the other hand, if $x \notin \operatorname{dom}(\alpha)$, then $x \mu \in \operatorname{im}(\mu) \backslash \operatorname{dom}(\alpha) \mu$, so that (by (4.1)) $x \mu \pi \in$ $X \backslash \operatorname{im}(\mu)$ and $x \mu \pi \mu^{-1}$ is undefined. Hence $\alpha=\mu \pi \mu^{-1} \in\left\langle\mathcal{S}_{X}, \mu\right\rangle$. Thus $\mathcal{I}_{X}=\left\langle\mathcal{S}_{X}, \mu\right\rangle$, as required.

Next, we determine the relative rank of $\mathcal{I}_{X}$ modulo $\mathcal{I E}_{X}$ the set of idempotents in $\mathcal{I}_{X}$. First, we record the following well-known and easily verified fact.

Lemma 4.6. The set of idempotents in the symmetric inverse semigroup is the set of all partial identity maps.

For details see [7, Theorem 5.1.5].

Theorem 4.7. The relative rank of $\mathcal{I}_{X}$ modulo $\mathcal{I E}_{X}$ is $\left|\mathcal{I}_{X}\right|$.

Proof. Let $A$ be any subset of $\mathcal{I}_{X}$ such that $\left\langle\mathcal{I E}_{X}, A\right\rangle=\mathcal{I}_{X}$. Then by Lemma 4.6 every element of $\left\langle\mathcal{I E}_{X}, A\right\rangle$ is a restriction of an element of $\langle A\rangle$. Since every element of $\mathcal{S}_{X}$ is not the proper restriction of any element of $\mathcal{I}_{X}$, it follows that $A$ must contain a generating set for $\mathcal{S}_{X}$. Thus $|A| \geqslant\left|S_{X}\right|=\left|I_{X}\right|$.

We conclude by stating the inverse semigroup analogue of [3, Theorem 5.8] (see Proposition 1.6) as an open question. It seems likely that the answer to this question is yes.

Open problem. Is it true that the relative rank of a subset $S$ of $\mathcal{I}_{X}$ is either uncountable or at most 1 ?

Acknowledgements. The authors gratefully acknowledge the contribution of the referee, whose detailed critique of the paper led to many improvements in the presentation. N.R. acknowledges partial support from INTAS 99/1224.

\section{References}

1. S. Banach, Sur un théorème de M. Sierpiński, Fund. Math. 25 (1935), 5-6.

2. T. Evans, Embedding theorems for multiplicative systems and projective geometries, Proc. Am. Math. Soc. 3 (1952), 614-620.

3. F. Galvin, Generating countable sets of permutations, J. Lond. Math. Soc. 51 (1995), 230-242.

4. T. E. HALL, Inverse and regular semigroups and amalgamation: a brief survey, in Proc. Symp. Regular Semigroups, Northern Illinois University (1979), pp. 49-78.

5. P. R. Halmos, Naive set theory, Undergraduate Texts in Mathematics (Springer, 1974).

6. G. Higman, B. H. Neumann and H. Neumann, Embedding theorems for groups, $J$. Lond. Math. Soc. 24 (1949), 247-254.

7. J. M. HowIE, Fundamentals of semigroup theory, London Mathematical Society, vol. 12 (Oxford University Press, 1995).

8. J. M. Howie, N. RuŠKuC And P. M. Higgins, On relative ranks of full transformation semigroups, Commun. Alg. 26 (1998), 733-748.

9. T. J. Jech, Set theory (Academic Press, 1978).

10. W. SiERPIŃSKI, Sur les suites infinies de fonctions définies dans les ensembles quelconques, Fund. Math. 24 (1935), 209-212. 